\title{
SPURport: Grid Portal for Earthquake Engineering Simulations
}

\author{
Tomasz Haupt, Anand Kalyanasundaram, Nisreen Ammari, Krishnendu Chandra, \\ Kamakhya Das, and Shravan Durvasula \\ Mississippi State University, Center for Advanced Vehicular Systems, \\ Box 5405, Mississippi State, MS 39762, USA \\ \{haupt, anand, ammari, krish, das, sharvan\}@cavs.msstate.edu \\ http: //www. cavs.msstate.edu
}

\begin{abstract}
This paper presents a successful implementation of the SPURport, a prototype Grid Portal for the earthquake engineering community. The portal eliminates the need for installing and maintaining simulation software by the end user. However, the portal infrastructure is application-neutral and can be used as a blueprint for developing portals for other communities. It provides seamless access to remote resources and supports the incorporation of legacy applications. It uses XML-based metadata extensively, which enables the introduction of high-level middle-tier services that aggregate and coordinate lowerlevel services provided by the Globus toolkit. For example, the high level Job Submission Service orchestrates resolution of logical entities, file transfers, and data streaming prior to actual job submission, hiding these activities from the end user.
\end{abstract}

\section{Introduction}

This paper describes a successful implementation of the SPURport - a prototype Grid Portal for the earthquake engineering community. The portal builds on and extends the current functionality of the NEESgrid [1], the cyberinfrastructure for the Network of Earthquake Engineering Simulations (NEES) [2]. The NEESgrid, in turn, is an application of OGSI/Globus Toolkit 3.0 [3]. The grid portal consists of three major components: a Web Portal providing the remote access to the SPURport functionality, the Grid Portal middleware that implements the portal specific services, and the backend comprising the computational resources as well as data and metadata repositories.

The Web Portal is implemented using the CHEF[4] portal toolkit that adds collaborative features to the JetSpeed[5] portlet framework hosted by the Apache-Tomcat server. Since the portal front end is implemented as a portlet, it can be implemented using other portlet containers. Indeed, we have an alternative implementation using JSR-169 compliant GridSphere[6] toolkit. The use of CHEF is dictated by the integration with the NEESgrid. The portlet technology is widely used to implement Web Portals and it is recommended by the Open Grid Computing Environments consortium [7]. The portal toolkits, such as CHEF or GridSphere, provide a set of build-in portlets such as secure login (including access to myProxy server), user management and templates for the portal pages layout. They also provide a support for accessing 
remote services through Globus infrastructure (Java Cog [8]). However, access to the low level Globus functionality is not sufficient for implementing complex Grid Portals such as SPURport.

The actual functionality of SPURport is implemented by the SPURport middleware that creates a bridge between the required portal functionality and low level Globus services. Particular important is support for the incorporation of the legacy applications and orchestrating Globus services to perform complex tasks. Although driven by the specific requirements of earthquake engineering, the middleware is applicationneutral, and can be reused for other application domains.

The rest of this paper is organized as follows: Section 2 briefly summarizes the goals of the SPUR project. Section 3 describes the requested functionality of the portal, and Section 4 explains the portal implementation. Due to space limitations, only selected portal features and the middle-tier implementation to support these features are discussed. Summary and conclusions are given in Section 5.

\section{Large-Scale Seismic Performance of Urban Regions}

The SPUR (Seismic Performance of Urban Regions) project is using the NEESgrid technology to link high-end simulations of earthquake ground motion, advanced models for building performance, large-scale databases, and visualizations to develop new knowledge about the spatial distribution of structural damage in a region and the effectiveness of building codes on controlling damage in a region. In this collaborative effort (between Mississippi State University, Carnegie Mellon University and Universities of California, Berkeley and Irvine) new visualization methods have been developed for understanding the dynamic processes in a large region, and new methods for communicating this information to scientists and engineers, as well as for conveying the effects of earthquakes to non-technical decision-makers. This is achieved through the use of the SPURport: a Grid-services-based middleware that provide access to computational resources, the databases for the regional models and simulations, as well as selecting scenarios and regional inventories for analysis.

\section{Functionality of SPURport}

The fundamental requirement for the SPURport is performing real time simulations of structural responses to ground motion caused by an earthquake. The implementation must provide an integrated simulation environment accessible anywhere through a Web Browser. Specifically, the portal must eliminate the need for explicit logging-in to remote systems (to retrieve, pre-process, transfer and post-process the data or submit jobs for execution); installation of any software; and the need for computerrelated knowledge of the end user. For example, the user might be not familiar with the operating system of the remote computer, the user might not know how to query metadata servers to find the data or interpret the XML-encoded response of the server, the user might not know how to transfer data from one remote location (data repository) to another (compute servers), the user might not know or understand the data format which is needed to extract and format input data for the simulations, the user might be not familiar with OpenSees framework and consequently might not know 
how to prepare and run the simulations, the user might not know how to verify the consistency of the results., i.e., making sure that the right data are fed to the correctly prepared OpenSees script, etc.

The SPURport is required to be an integrated simulation environment; to provide an easy access to all services needed to specify, configure and perform simulation, as well as access the simulation results without the need of leaving the Web Browser. Furthermore, the portal should provide access to other NEESgrid services such as data and metadata repositories thus enabling collaborations between the users, data sharing and data dissemination to interested parities. The portal must be fault tolerant in the sense that work done by the end user cannot be lost as a result of interruption of connectivity (voluntary or not). At any time, the user should be able to reconnect to the servers and resume the broken session. Finally, the user should be able to reconstruct all circumstances that led to any particular simulation performed through the portal.

The context for a simulation is determined by three elements: (1) the earthquake model, (2) the structure model and (3) the inventory of structures. The earthquake model (or fault type) defines the ground motion. The simulations of earthquakes have been performed by our collaborators at Carnegie Mellon University [9]. The SPURport provides access to a repository of the results of these simulations. The structure models, as well as the OpenSees-based structure simulation framework, have been provided by our partners at University of California Berkeley [10]. An inventory of structures is defined as a collection of structures of a given types (for example, buildings of different sizes constructed using different materials according to specific building codes) distributed according to a selected pattern over the region of the simulated earthquake. The result of simulations of the seismic performance of the inventory is a distribution of potential damages in the region caused by an earthquake. At this stage of this project, the statistical analysis of the inventory performance has been done off-line because it is computationally very expensive and thus cannot be done interactively. Instead, the SPURport provides access to the repository of the results. We anticipate a parallel version of OpenSees running on high performance platforms to be developed by UC Berkeley outside this project. Once it is available, the simulations of the inventories will be incorporated into the SPURport.

Currently, a typical use of the SPURport is as follows. The user, guided by the metadata viewer and interactive visualizations, selects an earthquake model. Similarly, the user selects a structure type and sets its parameters, creating an instance of the structure to be simulated interactively. Next, the user chooses an inventory type and gets access to pre-computed results of the inventory simulation for the selected earthquake model. Finally, the user places the previously defined structure instance at a desired location on the distribution plot and runs the real-time simulation. The value of this scenario is that the user may try different what-if cases by placing different kinds of structures at locations known from the inventory simulations to be high- or low-risk locations.

\section{SPURport Implementation}

The Front End of SPURport is a CHEF teamlet, and thus it is seamlessly integrated with the rest of the NEESgrid user interface. This integration makes it possible to directly use NEESgrid/Chef user authentication mechanisms. The SPURport user interface is implemented as a single applet. 
Figure 1 shows the layout of the SPURport GUI. The four text fields in the upper part of the SPURport applet display all currents selections that the user has made: Earthquake model, Structure Model, Inventory and Simulation (here, apparently at the beginning of a user session, only the Earthquake Model is selected). The rest of the applet frame is taken by the Swing Tabbed Pane: each tab corresponds to a particular function. The tabs are described in the following sections.

Access to Ground Motion Data Repository. Access to shared data repositories is a common use pattern for portals. SPURport provide support for this functionality through a combination of three web services: file repository service, metadata service and replica locator service. These three services are coordinated by a high-level data service provider, referred to as the data service façade. Each data set is uniquely identified by a logical Uniform Resource Identificator (URI), or a logical name, and is associated with its descriptor - a metadata record. The Replica Locator service resolves the URI into the physical location of the file - its Uniform Resource Locator (URL). Note that in general there is a one-to-many relationship between the data set URI and URL allowing for the creation file replicas to optimize access to it. This design follows a pattern employed by many other Grid-related efforts, and the implementation of the Replica Locator service is a part of the Globus distribution.

The typical use scenario is that user queries the metadata service to identify the data set, and the SPURport front end provides a graphical interface for making these queries and examining their results, as shown in Fig. 1). Once the file is selected, its

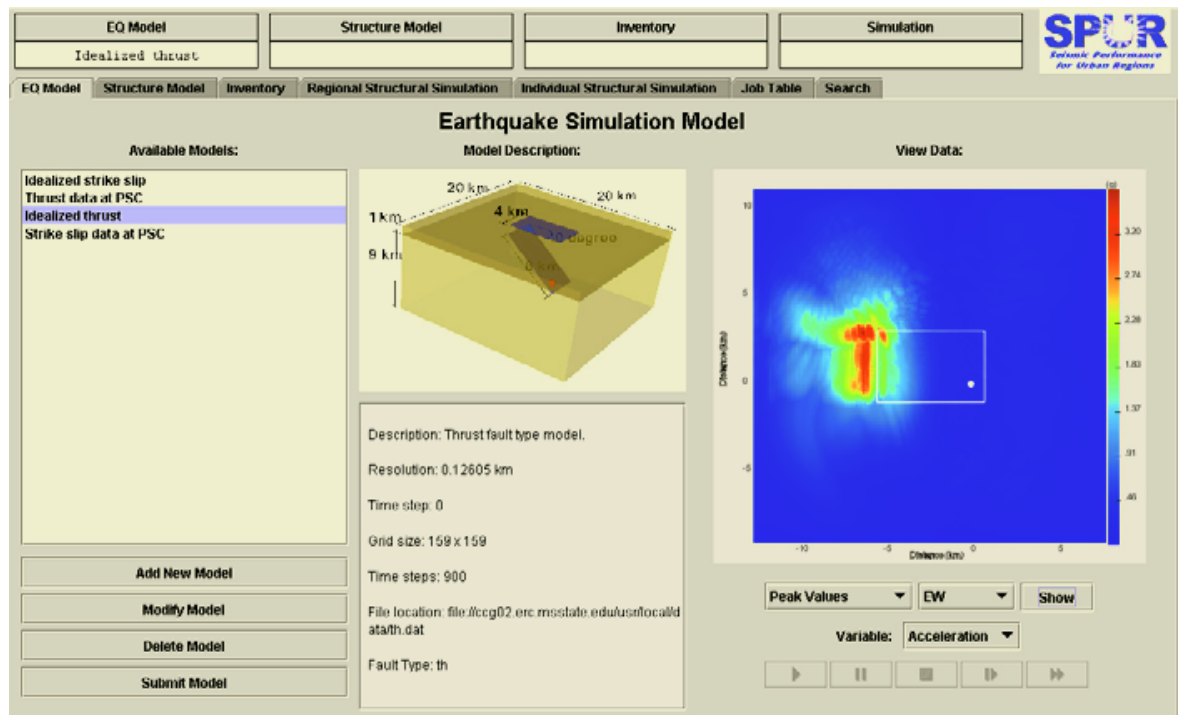

Fig. 1. SPURport GUI: Earthquake Model selection. The left panel shows a list of available datasets, the middle panel shows the metadata for the selected data set, and the right panel displays the contents of the retrieved file 
URI is resolved by the replica locator and the resulting URL of the data set is used to actually retrieve the data. To simplify the front-end implementation and to reduce communication overhead, the interactions between the services are orchestrated by the façade. Thus, once the URI of the file is known, it is just a single request made by the applet to initiate the file transfer to the selected location, or streaming it to the applet for visualization. The applet call is converted by a JSP to an invocation of a corresponding façade's method that in turn makes all necessary calls to the services, as needed. The façade accesses the services through adapters, making it unaware and thus independent from the actual implementation of the back-end services. The current SPURport uses NEESgrid's OGSI/GT3-compliant NMDS [11] as metadata service and flat file system accessible through GridFTP as a file repository.

Incorporation of a Legacy Application. The SPURport simulation capabilities are based on the OpenSees framework, a Grid-unaware, legacy application. It has been brought to the portal using a similar approach as used in our previous portals, Gateway and Distributed Marine Environment Forecast System [12]. The key concept here is the employment of a metadata that describes the application. This metadata is referred to as "application descriptor". The application descriptor is an XML document comprised of three parts: application signature (name, credits, support, and the like), description of the application parameters including i/o specification, and instructions on how to run it (e.g., location of the executables, values of environmental variables, etc) that is automatically translated by the SPURport Job Submission Service into the Globus Resource Specification Language (RSL) at the submission time. This is the SPURport mechanism of hiding the complexity of the running applications in the Grid environment. Incorporating OpenSees, and other legacy applications, into the portal is accomplished by creating the XML application descriptor by filling out a web form. This is a task for the application expert who understands the application requirements (hardware, environment, input data, application parameters, etc) and thus not the end user. The support for the application metadata is provided by the SPURport Metadata Service.

Job Configuration - Structure Models. OpenSees applications, as many other applications, are controlled by input files. In the case of OpenSees, the controlling input file is written in the OpenSees script (an extension to the Tcl scripting language). In particular, the geometry and properties of the structures to be modeled are defined using the OpenSees scripts. The SPURport provides a library of such scripts, maintained by the data repository (in addition to files representing ground motion). Consequently, the user can browse the metadata to select and upload the script that represents the structure of interest. Furthermore, OpenSees scripting support parameters, and therefore the properties of a particular structure type can be adjusted. These parameters are captured in the corresponding script's metadata. The SPURport user selects thus a structure type (OpenSees script), and the portal seamlessly downloads the script's metadata and generates in-the-fly a graphical user interface revealing all adjustable parameters for the selected type of structure. The user sets the values of these parameters (or leaves the default values) and saves them as an instance of the structure. These values are used by the Job Submission Service. 
Job Submission. The Globus toolkit provides all necessary services for submitting jobs on remote systems and the Java COG provides Java interfaces to these services making the invocation of the Globus services easy in the portal environment. However, the complete process of the job submission is a multistep process requiring coordination of several services. Therefore SPURport introduces its own, high-level Job Submission service that acts as a façade for the low-level Globus services and interacts with other SPURport services.

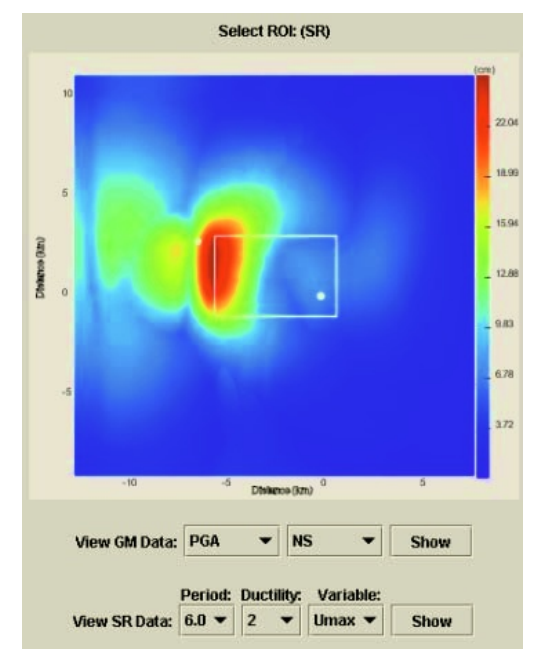

Fig. 2. To select the structure location the user displays the ground motion data or looks at a statistical distribution of previously run simulations. Here, the panel shows the distribution of $\mathrm{U}_{\max }$ (maximum displacement of the top most floors) for the selected period of structure oscillations and ductility. The white rectangle in the central part of the plot shows the location of the fault, and the white dot inside the rectangle shows the epicenter of the simulated earthquake. The user selects the location by clicking on the panel (c.f. the second white dot on top of a dark region corresponding to high $U_{\max }$ values, left of the fault, which resulted by placing the cursor in that spot and clicking the left mouse button)

To submit a job, a user environment for running the job is created. This involves creating a working directory on the target system. Then input files are staged to the working directory. The OpenSees simulations require two input files. One is the driving OpenSees script that must be retrieved from the data repository and modified as specified by the user (changes of the structure parameters). The other is the acceleration time-history of the ground motion at the location where the simulated structure is located (see Fig. 2). To get this file, the data corresponding to the selected earthquake model in the data repository are fed to a filtering routine that extract data points needed for the simulation. That pruned data set is transferred to the target machine. Next, the job submission request is created as the Globus RSL document by combining the information stored in the application descriptor, parameters set by the user as well as information about the user environment. At this moment the job is ready for submission, and the history metadata is created, so that a record on how the 
simulation was run is preserved for a future reference. Then the job is submitted through Globus GRAM and the Job Service registers itself as a target for GRAM notifications. All changes in the job status reported by GRAM are forwarded to the SPURport monitoring services. Once the job is completed, its output files (including stdout and stderr) are staged out to the location specified by the user.

Monitoring Job Status and Access to the Results . All users jobs submitted through the SPURport are accessible through the Job Table interface (Fig. 3), until explicitly deleted by the user. The table displays where and when the job was submitted and its current status (PENDING, RUNNING, DONE). Once the job is completed the user can query its properties (e.g., input files, values of parameters, and history) and access - view or download - its results, including standard output, standard error and output data files.

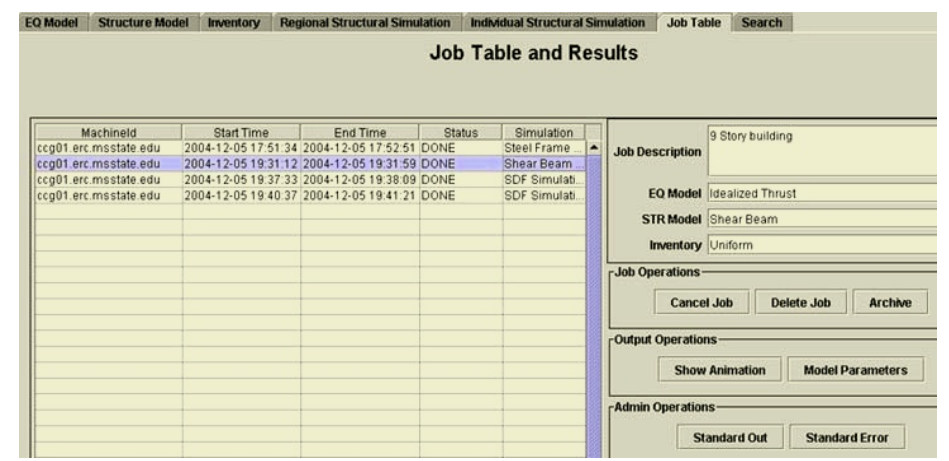

Fig. 3. SPURport Job Table interface allows monitoring the status of submitted jobs and provide access to their results. The jobs can be searched using metadata queries

\section{Conclusions}

This paper presents a successful implementation of a prototype Grid Portal for the earthquake engineering community. However, only the front-end has been customized for the particular domain. The portal infrastructure is application neutral and can be used as a blueprint for developing portals for other communities. It provides seamless access to remote resources (CPU and data) through OGSI/Globus 3.0 and provides support for easy incorporation of legacy applications. It extensively uses XML-based metadata for describing both data and applications. The metadata are used for automatic generation of the GUI, supporting the user with selection of applications and data. Furthermore, describing the application by metadata opens the opportunity to introduce the high-level middle-tier services that aggregate and coordinate lower-level services provided by the Globus toolkit.. Consequently, all the complexity of the heterogeneous, distributed Grid environment is hidden from the user. The applications and data are installed, maintained and upgraded by application experts. Therefore, the end user - the domain expert - can again concentrate on his or her research rather than acquiring computer wizardry. 


\section{Acknowledgements}

This work presented in this paper is a part of the NSF-sponsored project Large-Scale Seismic Performance for Urban Region (grant EEC0121989). We would like to thank our collaborators, in particular Gregory Fenves and Jaesung Park of UC Berkeley, and Jacobo Bielak of Carnegie Mellon University for invaluable discussions and help with designing the functionality of the SPURport, as well as providing us with the ground motion data and structural models. Last but not least, we would like to thank the SPUR project Principal Investigator, Roger King of Mississippi State University for coordinating this successful collaborative effort.

\section{References}

1. NEESgrid, http://www.gridnees.org

2. NEES, http://www.nees.org

3. Globus Project, http://www.globus.org

4. Chef, http://chefproject.org

5. JetSpeed, http://portals.apache.org/

6. GridSphere, http://www.gridsphere.org

7. Open Grid Computing Environments, http://www.collab-ogce.org/nmi/index.jsp

8. Java CoG, http://www.globus.org/cog

9. J. Bielak et al, "Visualizations of Large-Scale Earthquake Simulations", in the Proceedings of Supercomputing ' 03

10. OpenSees, http://opensees.berkeley.edu

11. NEESgrid Metadata Services, http://neesgrid.org/documents/TR_2004_38.pdf

12. T. Haupt, M. Pierce, "Distributed object-based grid computing environments", book chapter in "Grid Computing: Making the Global Infrastructure a Reality", Fran Berman, Geoffrey Fox and Tony Hey eds, John Wiley and Sons, 2003. 